\title{
LA NOZIONE GIURIDICA DI TERRITORIO
}

\author{
Maria Agostina Cabiddu
}

SUMARIO: I. IL TERRITORIO COME BENE GIURIDICO.- II. L'IMMATERIALITÀ DEL «BENE TERRITORIALE».— III. SEGUE: E LA SUA «PUBBLICITÀ».

\section{IL TERRITORIO COME BENE GIURIDICO}

Come sa chiunque, per una volta, abbia aperto un manuale di diritto pubblico, il territorio costituisce elemento fondante del fenomeno giuridico. II suo ruolo è però mutato nel corso della storia (1) e se nello Stato assoluto - in cui tutto il potere sovrano si concentrava nella persona del monarca (2) esso costituiva essenzialmente patrimonium principis, nel corso dell'Ottocento, con l'affermarsi dello Stato di diritto, divenne misura del potere, elemento identificatore dello stato nazionale in quanto stato territoriale e cioè Stato che aveva nel territorio non solo il limite della propria sfera di azione (sia verso gli altri stati sia al suo interno) ma anche una sua qualificazione, come soggetto legittimato in via esclusiva all'esercizio del potere in quell'ambito spaziale (3). Ciò

(1) Sul nesso territorio/diritto, C. SCHMITt, Der Nomos der Erde im Völkerrecht des Jus Publicum Europaeum (1974), trad. it, Milano, 1991. Ricostruisce il concetto e l'evoluzione del termine «territorio», dal punto di vista storico, L. MANNORI, La nozione di territorio fra antico e nuovo regime. Qualche appunto per uno studio sui modelli tipologici, in M. CAMMELL (a cura di), Territorialità e delocalizzazione nel governo locale, Bologna, 2007, 43 ss.

(2) Con il che non si intende affatto ignorare tutta quella storiografia che stigmatizzando le semplificazioni a cui, soprattutto i giuristi, troppo facilmente ricorrono nell'intento di sintetizzare fenomeni storici lunghi e complessi, sottopone a penetrante critica le nozioni di «stato assoluto», stato moderno», «stato». Peraltro, accolto o respinto come concetto dal pensiero politico e giuridico e dalla storiografia relativa, lo Stato rimane nei fatti europei occidentali dei secolo XVI-XVIII — quindi Stato moderno - un fenomeno di amministrazione pubblica senza precedenti per dimensioni e caratteristiche, meritevole per ciò solo di considerazione scientifica. In tal senso, E. ROTELL, Amministrazione e Costituzione. Storiografie dello Stato, Bologna, 2008.

(3) Ricostruisce in modo efficace e suggestivo la parabola dello stato nazionale, F. BENVENUTI, Dalla sovranità dello Stato persona alla sovranità dell'ordinamento, Relazione tenuta alla camera di commercio di Milano $\left(1^{\circ}\right.$ dicembre 1994), in Jus, 1995, II, 193 ss. Sul diritto come unità di ordinamento e di localizzazione, C. SCHMITT, Der Nomos der Erde im Völkerrecht des Jus Publicum Europaeum (1974), trad. it, Milano, 1991 
permise di concepire il territorio come elemento costitutivo dello persona giuridica statale, allo stesso modo del popolo che vi era insediato, neutralizzando le persone e la terra, degradate ad oggetto e luogo sul quale e nel quale esercitare la potestà sovrana (4).

Oggi, di fronte a un mondo senza confini, quale è quello della comunicazione e dell'economia - il mondo "globalizzato» -, i fondamenti teorici della territorialità intesa come garanzia della generalità rispetto agli interessi particolari delle persone e dei gruppi, e in quanto tale presupposto della sovranità e della rappresentanza politica, sono progressivamente erosi (5) e mutano di conseguenza i risvolti pratici attinenti all'organizzazione e all'azione dei pubblici poteri (6), se è vero che i medesimi processi a diffusione mondiale mettono in moto cambiamenti cruciali non solo nei rapporti tra gli stati ma anche all'interno degli stati (7). Dall'uniformità/omogeneità, finora assicurata dalla finzione dello Stato-persona (8), si passa infatti a un sistema policentrico in cui l'unità non è più un dato ma un obiettivo che si realizza, volta per volta,

(4) Sangue e terra, sono stati, come è noto, anche i presupposti della cittadinanza, sulle cui dottrine, v., per tutti, M. CUNIBERTI, La cittadinanza. Libertà dell'vomo e libertà del cittadino nella costituzione italiana, Padova, 1997.

(5) Sarebbe però errato parlare di dissoluzione della forma di Stato, secondo A. CARRINO, II diritto delle comunità locali tra processi di globalizzazione e crisi della sovranità, in Studi in onore di Giorgio Berti, I, Napoli, 2005, 648 ss. Il che, dal punto di vista del fenomeno giuridico, non smentisce il superamento della tesi ottocentesca che tendeva a sovrapporre diritto e Stato, con la precisazione che il «diritto degli spazi (...) non assorbe né annulla del tutto il diritto del territorio» secondo N. IRTI Norma e luoghi. Problemi di geo-diritto, Roma-Bari, 2001, 85 s.

(6) Al riguardo, lucidissimo, come sempre, G. GUARINO, Il governo del mondo globale, Firenze, 2000. Ma v. anche S. CASSESE, Oltre lo Stato, Roma-Bari, Laterza, 2006 e, naturalmente, F. BENVENUTI, II nuovo cittadino, Venezia, 1994

(7) I termini Glo-cal (globale/locale) e glocalization, proposti da A. MAYR ed elaborati da E. SWINGEDOUW (The Mammon Quest. "Glocalization», Interspatial Competition and the Monetary Order: The Construction of New Scales, in M Dunford-G. KafKalAs (a cura di), Cities and Regions in the New Europe, London, 1992, spec. 40 e 61), per quanto talmente abusati da risultare difficilmente riconducibili all'originario rigoroso significato analitico, alludono alla combinazione dei processi di globalizzazione e di riconfigurazione locale-territoriale, che sono il frutto dell'estensione dei rapporti economici - e, correlativamente, di sistemi normativi e di valore che li possano garantire - al di là dei confini nazionali, con le promesse e le minacce ad essa connesse, e la riscoperta delle «virtù» locali. Sul punto, v. gli studi di S. SASSEN, The Global City: New York, London, Tokyo (1991), trad. it., Torino, 1997; ID., Losing Control. Soveraignty in an Age of Globalization, New York, Columbia University Press, 1995. Sui cambiamenti istituzionali che investono anche il sistema delle autonomie locali, v. M. CAMmELL (a cura di), L'innovazione fra centro e periferia. Il caso di Bologna, Bologna, 2004. Sui rapporti fra globalizzazione e istituzioni, M.R. FERRARESE, Le istituzioni della globalizzazione, Bologna, 2000.

(8) Che in un mondo non più eurocentrico, dominato totalmente dall'organizzazione economico-scientifico-teconologica, sia messa radicalmente in pericolo la stessa civiltà giuridica sviluppatasi intorno a quell'idea di stato lo aveva visto bene C. SCHMITT, Der Nomos der Erde, cit. 
per via procedimentale e/o consensuale, attraverso nuove forme di rappresentanza e di decisione (9). Né sembrano rispondere efficacemente alle sfide del mutamento le pratiche dei governi locali, ostacolate da definizioni obsolete dei confini amministrativi - comuni, province, regioni (10) - che continuano a riflettere un'organizzazione territoriale in buona parte ormai inadeguata rispetto alle realtà insediative contemporanee, incapaci come sembrano di afferrare la dimensione della città infinita (1 1), delle grandi e anonime periferie dove la campagna si indurisce nella città (12), degli insediamenti rurali attraversati dalle grandi reti di comunicazione e non più distinguibili dal punto di vista socio-economico dai centri urbani, dei piccoli borghi abbandonati o ridotti a villaggi-vacanze (13).

In questo quadro, ben si comprende come il territorio non corrisponda più, necessariamente, all'insieme delle istituzioni e degli interessi localmente situati, né esaurisca il proprio significato nell'essere parte costitutiva degli enti c.d. appunto territoriali (14). La semantica del termine allude oggi piuttosto, anche nel comune sentire, a una nuova forma di rapporto fra l'individuo e il contesto ambientale che lo circonda e nel quale si svolgono le sue attività, una sorta di

(9) Pressoché sterminata, specie dopo la revisione del Titolo V della Costituzione, la letteratura su ruolo, modelli delle autonomie, strumenti di raccordo istituzionale negli ordinamenti multilivello. Sul punto, oltre ai molti volumi - fra i quali, recente, M. SCUDIERo (a cura di), Le autonomie al centro, Napoli, 2007 - basti consultare le ultime annate delle Riviste giuspubblicistiche e i siti - oltre quello che qui ci ospita - quali www.associazionedeicostituzionalisti.it; www.diritto-amministrativo.org; www.astrid-online.it; www.grupposanmartino.it.

(10) Sui paradossi delle riforme degli enti c.d. locali, v. E. ROTELL, Le aree metropolitane in Italia: una questione istituzionale insoluta, in G. MARTINOTTI (a cura di), La dimensione metropolitana, Bologna, 1999, 299 ss., ma v. anche, recente, G. MELIS, Introduzione: le trasformazioni del reticolo amministrativo nel Novecento: dalle Province alle «reti», in M. CAMmell (a cura di), Territorialità e delocalizzazione nel governo locale, cit., 123 ss.

(11) Fenomeno delle cui implicazioni si sono resi conto per primi sociologi e geografi piuttosto che giuristi e urbanisti. Sul punto, A. BONOMI - A- ABRUZZESE, La città diffusa, Milano, 2004.

(12) Mentre nel mondo la popolazione urbana ha ormai superato quella rurale, i confini tra città e campagna si attenuano, fino ad annacquarsi in un continuum di insediamenti abitativi, industriali, di consumo, di svago: la città non ha più bordi definiti, l' "urban sprawl» consuma il territorio, $\mathrm{i}$ «non luoghi» diventano i centri delle nuove città (v., ad es., le c.d. "centralità» della campagna romana), così come lo schermo televisivo è l'attuale focolare domestico. Sul punto, M. SORKIN, Variatons on a Theme Park. The New American City and the End of Public Space, New York, Noonday, 1994. Con specifico riferimento alla realtà milanese, S. BOERI, A. LANZANI, E. MARINI, II territorio che cambia. Ambienti, paesaggi e immagini della regione milanese, Milano, 1993.

(13) Sui profondi mutamenti che caratterizzano la fase più recente dell'urbanizzazione, v. il lavoro collettaneo curato da G. MARTINOTTI, La dimensione metropolitana, cit. e, in particolare, I'Introduzione.

(14) Cfr. il recente contributo di C. BARBATI-G. EndRICI, Territorialità positiva. Mercato, ambiente e poteri subnazionali, Bologna, 2005 e di M. CAMmell (a cura di), Territorialità e delocalizzazione nel governo locale, cit.. 
identità territoriale (15), che supera le categorie tradizionali della proprietà e del possesso e fa riferimento a una serie indefinita di interessi, cioè di beni, caratterizzati dall'essere parti di questo legame fondamentale e che si possono raccogliere - con una sintesi verbale che non ha una rispondenza nell'ordinamento positivo in senso stretto - nella nozione di «bene territoriale» (16).

Con il che, ancora non è chiaro se tale communis opinio si traduca in una categoria rilevante dal punto di vista giuridico o non si tratti piuttosto di una connotazione di valore generico, che, pur ponendosi in rapporto di genere a specie rispetto ad altre (giuridicamente significative) - quali «bene ambientale», «bene paesaggistico», «bene urbanistico», «bene infrastrutturale», etc. (17) - , di per sé non sia in grado di andare oltre la soglia del pregiuridico.

\section{L'IMMATERIALITÀ DEL «BENE TERRITORIALE»}

Proprio al fine di tentarne una ricostruzione giuridica, si può innanzitutto dire - ricalcando il ragionamento sviluppato, a suo tempo, da un illustre studioso per la categoria dei beni culturali (18) - che quella di «bene territoriale», come espressione di un nuovo rapporto dell'vomo con la realtà circostante, è nozione aperta, alla quale il diritto non dà un proprio contenuto definitorio, rinviando piuttosto ad altri campi del sapere: economia, politologia, sociologia, scienze naturali, etc. Come tale, essa risulta particolarmente idonea a rappresentare quella sensibilità, sviluppatasi negli ultimi due decenni, verso temi che hanno a che fare in maniera più o meno diretta con il territorio e le sue trasformazioni e con la crescente aspirazione al godimento e alla fruizione pubblica di beni — salute, ambiente, mobilità, energia, paesaggio,

(15) Sulle spinte contraddittorie che animano tali nuove identità fatte di appartenenza non meno che di sradicamento, v., per i profili giuspubblicistici, fra i primi, S. SICARDI, Essere di quel luogo. Brevi considerazioni sul significato di territorio e di appartenenza territoriale, in Pol. Dir., 2003.

(16) Risulta perciò evidente come l'urbanistica non tanto persegua il soddisfacimento di questo o quell'interesse - la cui individuazione e tutela forma oggetto di diverse discipline di settore - quanto piuttosto l'equilibrio fra tutti i possibili interessi che insistono su un certo territorio e possono interferire con il suo assetto. Cfr.. P. Stella RiCHTER, Profili funzionali dell'urbanistica, Milano, 1984, passim e F. BENVENUTI, Pianificazione del territorio e tutela del cittadino, in Jus, XXXIV, 1987, II, 135 ss.

(17) Peraltro, che tali beni rappresentino altrettanti settori del governo del territorio, oltre che oggetto di specifica disciplina, sembra essere un dato del tutto acquisito. Basti il riferimento ai saggi di P. URBANI, Governo del territorio e luoghi di vita e di lavoro; G. CAIA, Governo del territorio e attività economiche; F. FRACCHIA, Governo del territorio e ambiente; P. Stella RICHTER, Governo del territorio e infrastrutture; S. Civitarese MatTeuccl, Governo del territorio e paesaggio, in S. Civitarese, E. Ferrari, P. Urbani (a cura di), Il governo del territorio, Milano, 2003, 183 ss.

(18) II riferimento è, ovviamente, a M.S. GIANNINI, I beni culturali, in Riv. trim. dir. pubbl., 1976. 
sviluppo, cultura, bellezza, etc. - , che segna il passaggio dal Welfare State allo stato del well-being o della good-life. Non solo: proprio per il suo essere suscettibile di continua evoluzione e di adattamento alle diversità di contesto e di situazione conferisce a tale nozione l'innegabile pregio di consentire la più ampia possibilità di intervento pubblico.

Ovviamente, ciascuna legge avente ad oggetto le singole specie di interessi, cui si è fatto sopra riferimento - l'urbanistica, la difesa del suolo, la protezione civile, la tutela e la valorizzazione dei beni culturali, le grandi reti di trasporto, i porti e gli aeroporti, la produzione e la trasmissione dell'energia, la gestione delle risorse idriche, lo smaltimento dei rifiuti, la salvaguardia dell'ambiente e dell'ecosistema, la tutela e la promozione dei prodotti locali, etc. - , ricostruisce a suo modo organizzazione, procedimenti, criteri di composizione di interessi e di misure adottabili (in una parola: disciplina), dando così vita a ordini normativi separati che non tengono conto di una evidente interdipendenza fra le esigenze rilevate. Eppure, non è difficile cogliere, al di là dell'eterogeneità dei beni e delle specificità delle normative di settore, l'elemento che, dal nostro punto di vista, tutte le accomuna, ovvero l'interesse oggettivo, riferibile a qualsiasi «entità del mondo esterno» in quanto portatrice del «valore territoriale» (19), come espressione, appunto, della fondamentale esigenza di equilibrio fra la protezione delle cose e lo sfruttamento delle stesse, in vista del benessere e del progresso economico e civile della comunità insediata su un determinato territorio (20). In effetti, se il perseguimento di un certo

(19) Sul rapporto fra urbanistica e interessi differenziati, cfr. V. CERULI IRELLI, Pianificazione urbanistica e interessi differenziati, in Riv. trim. dir. pubbl., 1985, 386 ss.

(20) Sul rapporto uomo-ambiente circostante come nucleo sostanziale della disciplina urbanistica, v. A. Predierl, Pianificazione e costituzione, Milano, 1963, che pionieristicamente colloca la pianificazione urbanistica «in un contesto di presupposti economici considerati dalla costituzione come quello su cui deve operare la regolazione dell'economia; e a quest'ultima la pianificazione urbanistica è normativamente accomunata, come strumento per la attuazione ed imposizione di fini sociali alla proprietà, e, in particolare delle finalità di trasformazione degli assetti socioeconomici esistenti ed inadeguati da modificare e sistemare in modo più razionale e più consono alle esigenze di giustizia sociale, di benessere, di dignità e di libertà della persona, migliorando il rapporto fra l'uomo e l'ambiente». Peraltro, se dal punto di vista logico, la pianificazione urbanistica - e, a maggior ragione, il governo del territorio - può considerarsi una specie della categoria «piano 0 programma economico», dal punto di vista cronologico, cioè storico, vale il contrario, giacchè vengono prima le specie e poi il genere. In realtà, come è stato rilevato (M.S. GIANNINI, Pianificazione, ad vocem, in EdD, XXXIII, Milano, 1983) le pianificazioni esistono da quando esiste l'azione amministrativa, ma, in quanto tecniche di azione, sono state, a lungo, ritenute attinenti al fatto non al diritto. Le prime specie di piani giuridicamente rilevanti si hanno nella contabilità pubblica e poi nelI'urbanistica, mentre quelle propriamente economiche compaiono molto più tardi, nel sec. XX, con gli Stati socialisti, dove costituiscono lo strumento per la direzione pubblica dell'economia. Sull'evoluzione del concetto e dei relativi strumenti, v., recente, N. RANGONE, Le programmazioni economiche. L'intervento pubblico in economia tra piani e regole, Bologna, 2007. 
interesse può portare alla distruzione o alla contaminazione di tutto ciò che I'attuale sensibilità culturale riconduce all'idea di territorio (21), I'assunzione di un punto di vista superiore, permette di cogliere l'interesse al «bene territoriale» unitariamente inteso, rappresentando nello stesso tempo la realizzazione di una funzione di polizia e di una funzione di impulso e direzione dello sviluppo (22). Solo in una visione complessiva e coordinata il territorio diventa, infatti, elemento attivo, e non quindi soltanto passivo e inerte, dell'organizzazione sociale (23).

Questo spiega non solo come qualsiasi entità del mondo esterno possa assumere o, viceversa, perdere valore territoriale - dal fabbricato residenziale, all'insediamento produttivo, dalla risorsa naturale alla localizzazione infrastrutturale - ma anche l'irriducibile ambivalenza che caratterizza l'oggetto del nostro discorso: da un lato, come già accennato, presupposto necessario per una più ampia e migliore tutela di interessi collettivi non altrimenti adeguatamente presi in considerazione dall'ordinamento, dall'altro, nodo di resistenze e di reazioni interdittive che, pur riconoscendo la bontà degli interessi più generali, finiscono spesso per interpretarli in chiave egoistica e di autoprotezione, opponendosi alle localizzazioni sgradite (discariche, elettrodotti, antenne, reti di trasporto, etc.). Sotto quest'ultimo profilo, il fenomeno c.d. della sindrome Nimby (not in my back yard) rappresenta in maniera emblematica non solo l'atteggiamento che consiste nel riconoscere come necessarie, o comunque possibili, opere e attività di interesse pubblico che, nel contempo, si dichiarano indesiderabili per via del temuto impatto «ambientale» negativo ma anche il difficile rapporto fra scienza e diritto, specie quando il diritto è chiamato a rivestire di certezza ciò che per la scienza risulta incerto (si pensi, per esempio, al caso dell'elettrosmog o allo smaltimento dei rifiuti) (24).

Di più. Spiega anche, e soprattutto, in che cosa consista il bene giuridico «territorio» quale si va qui delineando. Questo, infatti, pur quando supportato da cose (terra, infrastrutture, opere, etc.), non si identifica con le stesse e con

(21) Affronta questi temi, da un punto di vista storico, P. BEVILACQUA, La terra è finita. Breve storia dell'ambiente, Milano-Bari, 2006.

(22) E. BosColo, Le regole per lo spazio urbano: dal piano regolatore alle politiche urbane, in S. Civitarese, E. Ferrari, P. Urbani (a cura di), Il governo del territorio, cit., 355 ss. Ma v. anche P. Stella Richter, Profili funzionali dell'urbanistica, cit., passim; F. SAlVIA e F. Teresl, Diritto urbanistico, VIl ed., Padova, 2002, 46.

(23) In questo ambito, la discrezionalità amministrativa, come ponderazione comparativa degli interessi, ha modo di esercitarsi al massimo livello, data la complessità dei profili che vengono in rilievo. Al riguardo, v. il recente contributo di L. CASINI, L'equilibrio degli interessi nel governo del territorio, Milano, 2005

(24) Sul punto, cfr. G. EndRICI, Territori e ambiente, in C. BARBATI-G. ENDRICI, Territorialità positiva, cit., 131 ss.). 
la loro materialità: un quartiere è certamente un aggregato di cose ed è pacifico che su una stessa cosa - e a maggior ragione su un complesso di cose - possano insistere più diritti: proprietà collettive (strade), pubbliche (scuole, uffici pubblici), private, condominiali, diritti d'uso e di abitazione, di garanzia, etc. Tutta questa varietà di posizioni giuridiche non riguarda, tuttavia, l'essere il quartiere, in quanto tale, un «bene territoriale», giacché questo non coincide con l'insieme degli edifici, delle opere e delle aree che compongono il quartiere ma è piuttosto un valore immateriale, che sorge dalle molte e varie cose naturali e artificiali che lo compongono e che sono comprese nel suo perimetro fisico, ma si distingue da esse, nel senso che esse ne costituiscono il supporto materiale ma non integrano il bene giuridico, che è - e rimane — immateriale. Come bene patrimoniale, il territorio può dunque dar luogo a rapporti in re o propter rem, formando oggetto del diritto di proprietà e/o di altri diritti; come bene giuridico in sé considerato esso soggiace invece a potestà pubbliche volte non a soddisfare un interesse personalizzato ma piuttosto a stabilire un rapporto che non è «sul» ma «per il territorio» (25), salvaguardando l'effettiva, non astratta, relazione di godimento che può costituirsi tra gli individui - a prescindere dal titolo formale - e la realtà esteriore (26). Basti pensare, da questo ultimo punto di vista, al fallimento degli standard urbanistici come disciplinati dalla legge n. 765/1967 e alle proposte di una loro attualizzazione sulla base di parametri di tipo non solo quantitativo ma anche qualitativo-funzionale (27), che sappiano tener conto, ad es., del differente grado di utilizzazione di immobili della medesima cubatura la seconda che siano destinati a residenza o a seconda casa), della domanda di mobilità (28), della incidenza su un'area della c.d. "popolazione attratta», cioè di

(25) Per questa precisazione, F. Benvenuti, Pianificazione del territorio e tutela del cittadino, cit., 137.

(26) Cfr., con riferimento ai beni culturali, G. PIVA, Cose d'arte, ad vocem, in Enc. Dir., XI, Milano, 1962.

(27) Sulla necessità di passare «dall'urbanistica meramente disegnata ad un'urbanistica reale», v. P. Stella Richter, Necessità e possibilità della pianificazione urbanistica, in F. Puglese - F. FerRARI (a cura di), Presente e futuro della pianificazione urbanistica, Milano, 1995, in part. 89, ma v. anche A. ChIERICHEtTI, Gli standard urbanistici qualitativi, in Governo del territorio, Atti del seminario del 15 novembre 2002, promosso dalla Consulta regionale lombarda e dall'INU Lombardia. Sul piano normativo, tra i primi interventi, v. la I.r. Lombardia, 15 gennaio 2001, n. 1, con la quale si è sostituita l'idea della capacità insediativa teorica con un'analisi dell'effettivo andamento demografico rapportato ai bisogni di spazio di ogni soggetto gravitante sul territorio, a prescindere dalla residenza anagrafica, e si è introdotto il c.d. piano dei servizi, documento che accede al p.r.g. e individua i livelli di soddisfacimento dei bisogni dell'utenza e i riflessi territoriali delle politiche dei servizi pubblici.

(28) Che il movimento e la circolazione (delle persone e delle merci) producano conseguenze sull'uso del suolo e sulla «densità» percepita della popolazione è cosa ovvia, per quanto non sempre adeguatamente considerata dai pianificatori. Sul punto, collegato al tema della crescente domanda 
coloro che - pur non risiedendo nella zona - ne usano abitualmente per lavoro, per studio, per bisogno o per svago - i servizi destinati alla collettività (29).

L'immaterialità del bene considerato risulta peraltro evidente non appena si ponga mente al fatto che le singole cose possono fisicamente mutare e persino perire, così come può mutare la loro condizione patrimoniale, senza che per questo venga meno il quartiere e le necessità della convivenza che ad esso si riferiscono e che formano oggetto del bene territoriale (30).

\section{SEGUE: E LA SUA «PUBBLICITÀ»}

Proprio a queste ultime si lega l'altro carattere del «bene territoriale», ovvero il suo essere bene pubblico (31). È appena il caso di precisare, dopo quanto detto, che la pubblicità non è qui da intendere in senso soggettivo, come appartenenza della cosa al demanio o al patrimonio dell'amministrazione ma piuttosto come inerenza del bene a un interesse generale, quello appunto di conservare le cose e il loro contesto pur secondando i processi vitali della società (32). Interesse particolarmente sentito oggi che la «territorialità» (33) è diventata parte integrante dell'agenda politica e «ragione

di spazio per usi non di produzione o di investimento ma di mero consumo, v. B. CoRI, La competizione per l'uso del suolo nelle aree metropolitane, in G. MARTINOTTI, La dimensione metropolitana, cit., 231 ss.

(29) Sul punto, G. MARTINOtTI (Metropoli. La nuova morfologia sociale della città, Bologna, 1993), che distingue le diverse popolazioni urbane (residenti, pendolari, studenti, turisti, ricoverati negli ospedali, etc.), riconoscendo a ciascuna di esse un peso specifico differente e un conseguente diverso impatto territoriale. V. anche P. NOBILE, Milano. Nuove forme di piano ... per quale città e per quali cittadini, in Urbanistica e informazioni, 2001, 176.

(30) Al mutare dei quartieri e delle città, in dipendenza delle trasformazioni economiche e sociali - basti pensare al passaggio dall'industria al terziario - corrisponde anche l'evoluzione delle politiche urbane, chiamate a orientare l'uso e il riuso degli spazi ma anche ad individuare strumenti diversi e più articolati per organizzare il territorio e la società. Sui rischi, per la sopravvivenza dell'identità dei luoghi, derivanti dallo smarrimento di una visione complessiva dei processi di trasformazione e, più profondamente, di un'idea di città e di territorio come un tutto unitario, v. recente, P. BERDINI, La città in vendita. Centri storici e mercato senza regole, Roma, 2008 e, con specifico riferimento al patrimonio culturale e al paesaggio italiani, come sintesi identitaria, S. SETTIS, Italia S.p.A.. L'assalto al patrimonio culturale, Torino, 2002.

(31) Con specifico riferimento all'urbanistica, v. recente, C. BIANCHETTI, Urbanistica e sfera pubblica, Roma, 2008.

(32) II passaggio dal «soggettivo» all'»oggettivo» ha caratterizzato, come noto, anche altri ambiti dell'amministrazione come organizzazione, a iniziare dai servizi pubblici. Sul punto, per tutti, U. Рототschnig, I pubblici servizi, Padova, 1964.

(33) Dovrebbe essere chiaro dopo quanto detto nel testo, ed è stato autorevolmente notato, che vi può essere una territorialità «negativa, che consiste nel potere di escludere altri soggetti dal 
sociale» di associazioni e comitati di cittadini che rapidamente sorgono quando l'ambiente, il paesaggio, il clima o un prodotto tipico sono messi in pericolo.

Dunque, sostantivo è il bene, non la cosa che ne può costituire, volta per volta, il supporto materiale, ed è facile comprendere come la posizione dell'ente pubblico, nella sua qualità di soggetto esponenziale della comunità, non tanto si qualifichi per la titolarità di diritti di natura patrimoniale quanto piuttosto per i poteri normativi e conformativi di cui esso si avvale per il perseguimento dell'interesse comune.

A ciò corrisponde, dalla parte del cittadino proprietario o imprenditore, una soggezione nei confronti dell'esercizio delle potestà pubbliche finalizzate alla tutela e alla valorizzazione del bene, che si traduce nell'essere destinatario di provvedimenti i cui effetti giuridici finiscono per interessare non solo il bene giuridico territoriale ma anche la res, ovvero ciò che ne costituisce il supporto fisico nei suoi profili patrimoniali (34). Basti pensare a tutta la problematica relativa all'estensione delle prescrizioni conformative del diritto di proprietà, connessa all'esercizio dei poteri pianificatori e, in particolare, all'apposizione dei vincoli urbanistici (35).

Sul piano più generale - e non è aspetto meno rilevante - al riconoscimento del territorio come bene giuridico corrisponde invece una posizione attiva, ovvero la pretesa del cittadino, in quanto tale, a partecipare, singolarmente e collettivamente, alle scelte circa la destinazione e l'uso del territorio, a partire dalla progettazione, fino alla decisione e all'attuazione, sul presupposto di una condizione paritaria dei diversi attori (pubblici e privati) e della loro appartenenza a uno stesso mondo, a uno stesso ordinamento (36), dove norme e attività si rincorrono e si compongono in atti, secondo criteri elastici e senza la pretesa di soluzioni definitive.

controllo di uno spazio geografico confinato; e una positiva, che consiste nel produrre valore (sociale, culturale, ambientale, economico) a partire dalle risorse potenziali specifiche di un territorio, mediante integrazioni sinergiche dei soggetti locali tra loro e con soggetti sovralocali» (G. DEMATTEIS E F. Governa, cit. in M. CAMmell (a cura di), Territorialità e delocalizzazione nel governo locale, cit., 9, n. 2.

(34) Sulla necessità di un corretto esercizio della discrezionalità, come capacità dell'amministrazione di individuare e ponderare tutti gli interessi in gioco, senza lasciarsene catturare, v. recente P. URBANI, Territorio e poteri emergenti. Le politiche di sviluppo tra urbanistica e mercato, Torino, 2007.

(35) Sul tema delle rendite edificatorie, v., fra gli altri, G. CORSO, Il controllo del mercato delle aree nella disciplina urbanistica, Palermo, 1971; F. SPANTIGATI, II governo della rendita fondiaria, in Jus, 1989, 41 ss.

(36) Cfr. L. MAZzA, Prove parziali di riforma urbanistica, Milano, 2004; P.C. Palermo, Prove di innovazione, Milano, 2001; S. MORONI, Pianificazione del territorio: ragioni, bisogni, responsabilità, Torino, 2001. 
Quanto ai rapporti fra cittadini, la pubblicità del territorio allude, com'è evidente, al suo essere bene comune $e$, in questo senso, bene non esclusivo - quando non addirittura inclusivo (37) - , capace cioè di fondare uno spazio sociale compatibile. A differenza dei beni materiali o esclusivi, la cui ricerca è alla base di relazioni sociali di mutua esclusione, secondo la regola per cui «dove sono io non c'è posto per te», il territorio come bene pubblico immateriale presuppone infatti un «noi» (38), una comunità della quale si è parte e nella quale ci si riconosce.

Al fondo del necessario equilibrio fra presenza uti singuli e presenza uti socii, la consapevolezza, secondo l'insegnamento dei saggi, che la terra non l'abbiamo ricevuta in eredità dai padri ma solo presa in prestito dai figli.

(37) Per la distinzione fra interessi/beni esclusivi, non-esclusivi e inclusivi, L. LOMBARDI VALLAURI, Corso di filosofia del diritto, Padova, 1981, 444 ss.

(38) Beni pubblici e beni privatii, Roma, 2005. 